\title{
Case of Urachal Cyst: A Rare Anomaly
}

\section{Dr. Bhavin Kothari}

Associate Professor, Department of Surgery, Gujarat Adani Institute of Medical Sciences, Bhuj, Gujarat, India

\begin{abstract}
Urachal abnormalities result from incomplete regression of the foetalurachus. They are more common in children than in adults, due to urachal obliteration in early infancy. A 32 year old male presented to surgical department with a 3 months history of foul smelling purulent umbilical discharge associated with constant lower abdominal pain, low grade fever without chills and rigors. A midline incision was used to excise the cyst, together with the omentum adherent to it posteriorly. Histopathological analysis of the resected specimen showed chronic inflammation with no evidence of malignancy. Urachal diverticulum seems to be the rarest of the anomalies in all series, with some recent series not reporting any. Urachal anomalies are rare in adults. Presentation is atypical; therefore, a high index of suspicion is required in order to achieve a diagnosis. A triad of lower midline mass, umbilical discharge and sepsis is suggestive, although MRI confirms the diagnosis and defines the surrounding anatomical relationship. Complete surgical excision is the treatment of choice due to the risk of malignant transformation. We recommend a 2 stage treatment with a combination of broad spectrum antibiotics or incision and drainage, followed by interval excision after resolution of sepsis.
\end{abstract}

Keywords: Anomalies, incision, MRI, Urachal

\section{Introduction}

Urachal abnormalities result from incomplete regression of the foetalurachus. They are more common in children than in adults, due to urachal obliteration in early infancy ${ }^{1}$. In adults, urachal cyst (UC) is the commonest variety, with infection being the usual mode of presentation ${ }^{2}$. Diagnosis remains challenging due to the rarity of this lesion and the non-specific nature of its symptomatology. Since the first description of urachal abnormality by Cabriolus in 1550 , few cases have been reported in literature. We describe a case of urachal cyst presenting with abdominal pain,foul smelling purulent umbilical discharge and sepsis, in a previously fit and well man.

\section{Case Presentation}

A 32 year old male presented to surgical department with a 3 months history of foul smelling purulent umbilical discharge associated with constant lower abdominal pain, low grade fever without chills and rigors. He gave no history of nausea, vomiting or change in bowel habit. Systemic review revealed no abnormality. He had completed a course of antibiotics prescribed by his General Practitioner with little relief.

On examination, he was apyrexial and haemodynamically stable. Abdominal examination, however, revealed purulent foul smelling umbilical discharge with minimal surrounding erythema and tenderness in infraumbilical region. A working differential diagnosis of omphalitis or pilonidal disease of the umbilicus, or patent urachus was made.

Haematology showed a raised C-reactive protein, but normal white cell count. Microbiology culture of the pus grew Bacteroides sp.

Abdominal ultrasound scan showed a $3.8 \mathrm{~cm}$ echogenic collection in a cavity within the anterior abdominal wall in the midline. An MRI scan, confirmed the diagnosis of UC communicating proximally with the umbilicus (Figure 1). The MRI scan was carried out on an full bladder making to exclude possible distal communication between the UC and the bladder.

The patient was initially treated with intravenous antibiotics for 5 days with surgery planned at the end of this period. A midline incision was used to excise the cyst, together with the omentum adherent to it posteriorly. Histopathological analysis of the resected specimen showed chronic inflammation with no evidence of malignancy. Recovery was unremarkable.

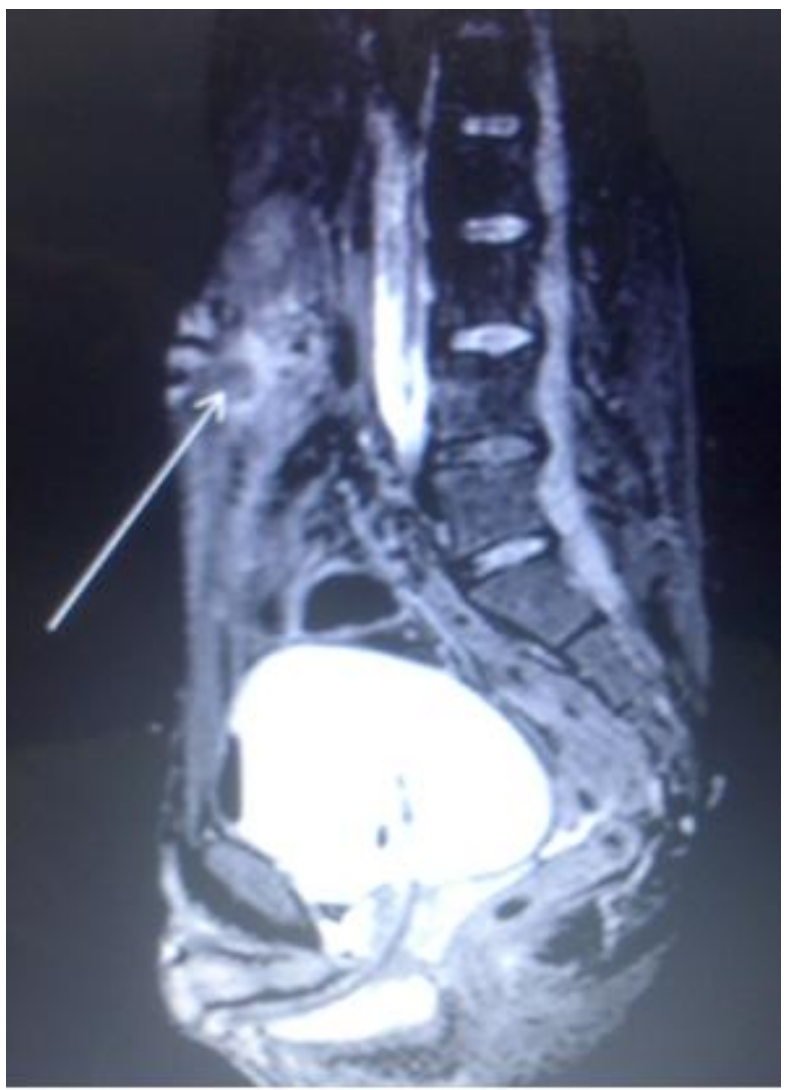

Figure 1: MRI scan showing high signal fluid within the umbilicus tracking into urachal remnant 


\section{International Journal of Science and Research (IJSR) \\ ISSN (Online): 2319-7064}

Index Copernicus Value (2013): 6.14 | Impact Factor (2014): 5.611

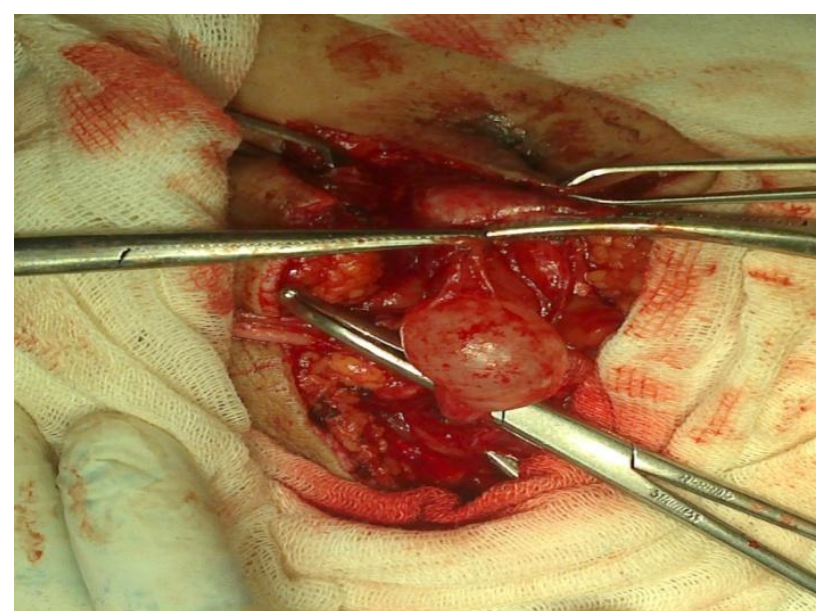

\section{Discussion}

\section{Embryology}

The urachus, developmentally is the upper part of the bladder, both of which arise from the ventral part of the cloaca ${ }^{3}$. Descent of the bladder from the $5^{\text {th }}$ month of development into the foetal pelvis pulls the urachus with it resulting in the formation of the urachal canal. The lumen of this canal progressively obliterates during foetal life, with eventual formation of a fibrous tract in early adult life.

Figure 2: Showing Intraoperative View of Urachal Cyst.
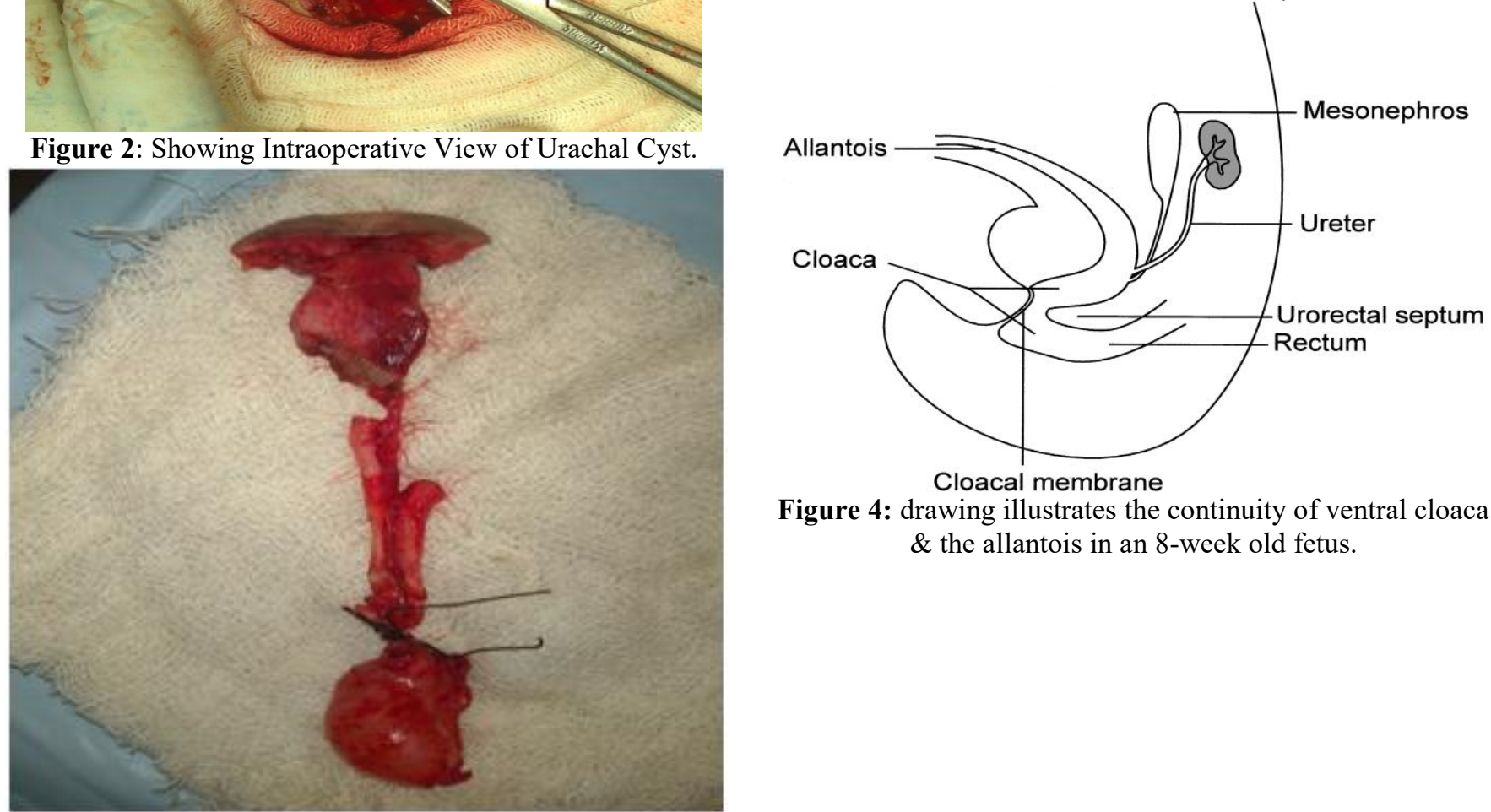

Figure 4: drawing illustrates the continuity of ventral cloaca $\&$ the allantois in an 8-week old fetus.

Figure 3: showing completely excised specimen of urachal cyst

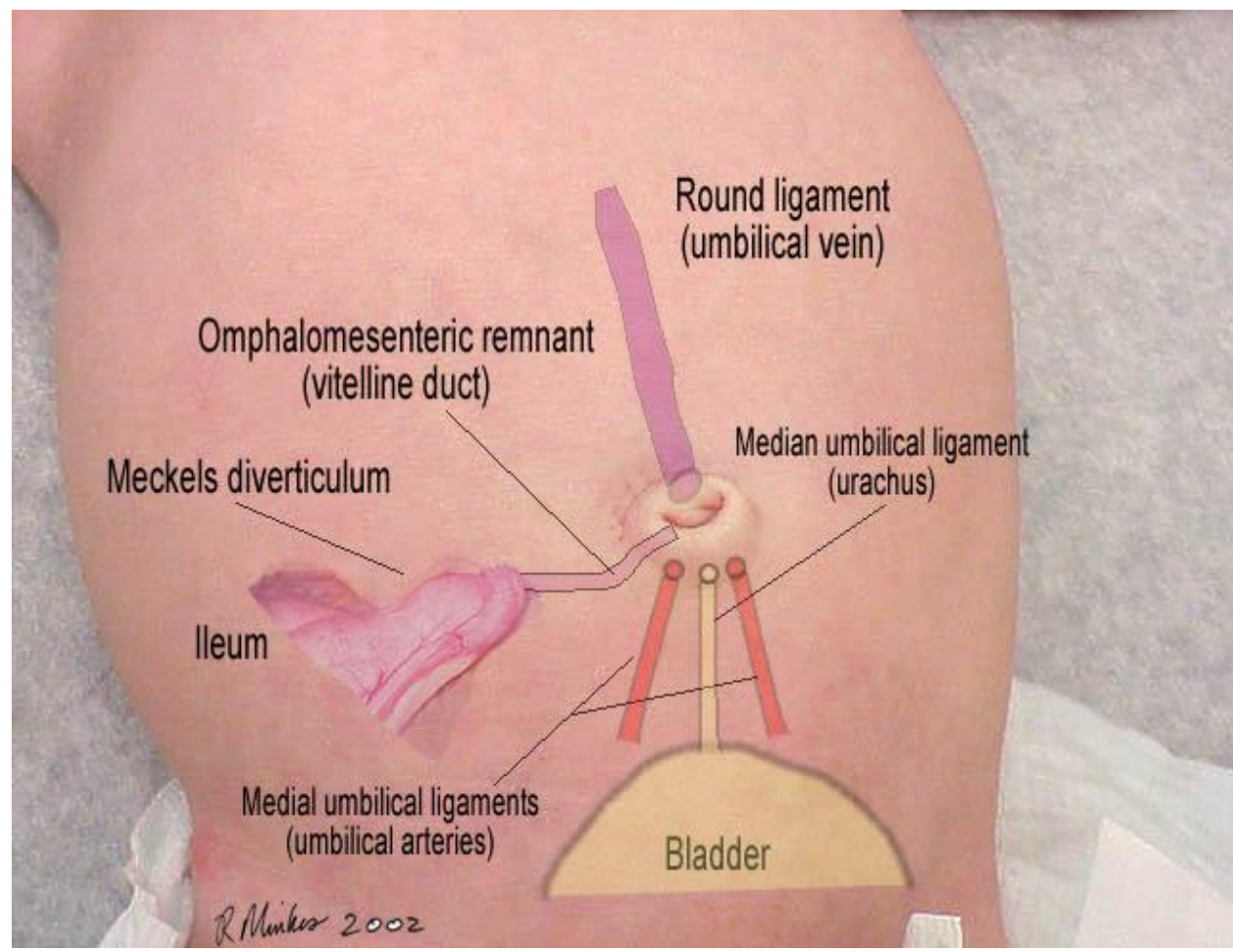

Figure 5: figure showing relations of urachus with umbilicus.

Volume 4 Issue 11, November 2015 www.ijsr.net 


\section{International Journal of Science and Research (IJSR) \\ ISSN (Online): 2319-7064 \\ Index Copernicus Value (2013): 6.14 | Impact Factor (2014): 5.611}

At the end of development, the urachus lies between the transversalis fascia anteriorly and the peritoneum posteriorly (space of Retzius), surrounded by loose areolar tissue and attaches the umbilicus to the dome of the bladder

Histologically, it is composed of 3 layers;

- an innermost layer of modified transitional epithelium similar to the urothelium,

- the middle layer of fibroconnective tissue and

- Outermost layer of smooth muscle continuous with the detrusor ${ }^{1,3}$

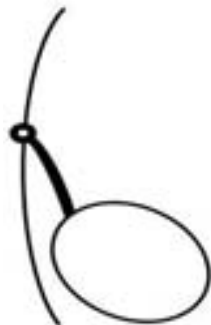

A

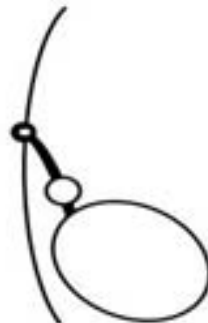

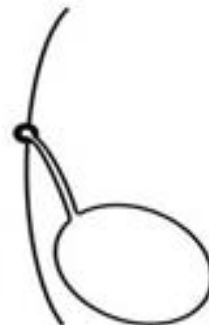

C

\section{Classification of Urachal Anomalies}

Urachal anomalies are due to failure of complete obliteration of the lumen during gestation [4]. Their anatomical classification is based on the degree to which the patency of the urachus has persisted. Typical urachal anomalies are shown in Figure 6. The possibilities vary from a completely patent urachus that allows urine to freely drain through the umbilicus to a small blind-ending sinus tract from the skin. Aurachal cyst may be present at any location along the length of the urachus but are most commonly found near the dome of the bladder. Aurachal diverticulum is a partial patency of the urachus draining into the dome of the bladder.

Figure 6: Diagram illustrating different types of urachal anomalies. A- Normal obliteration of the urachal lumen. B- Urachal cyst. C- Patent urachus. D- Urachal sinus. E- Urachal diverticulum.
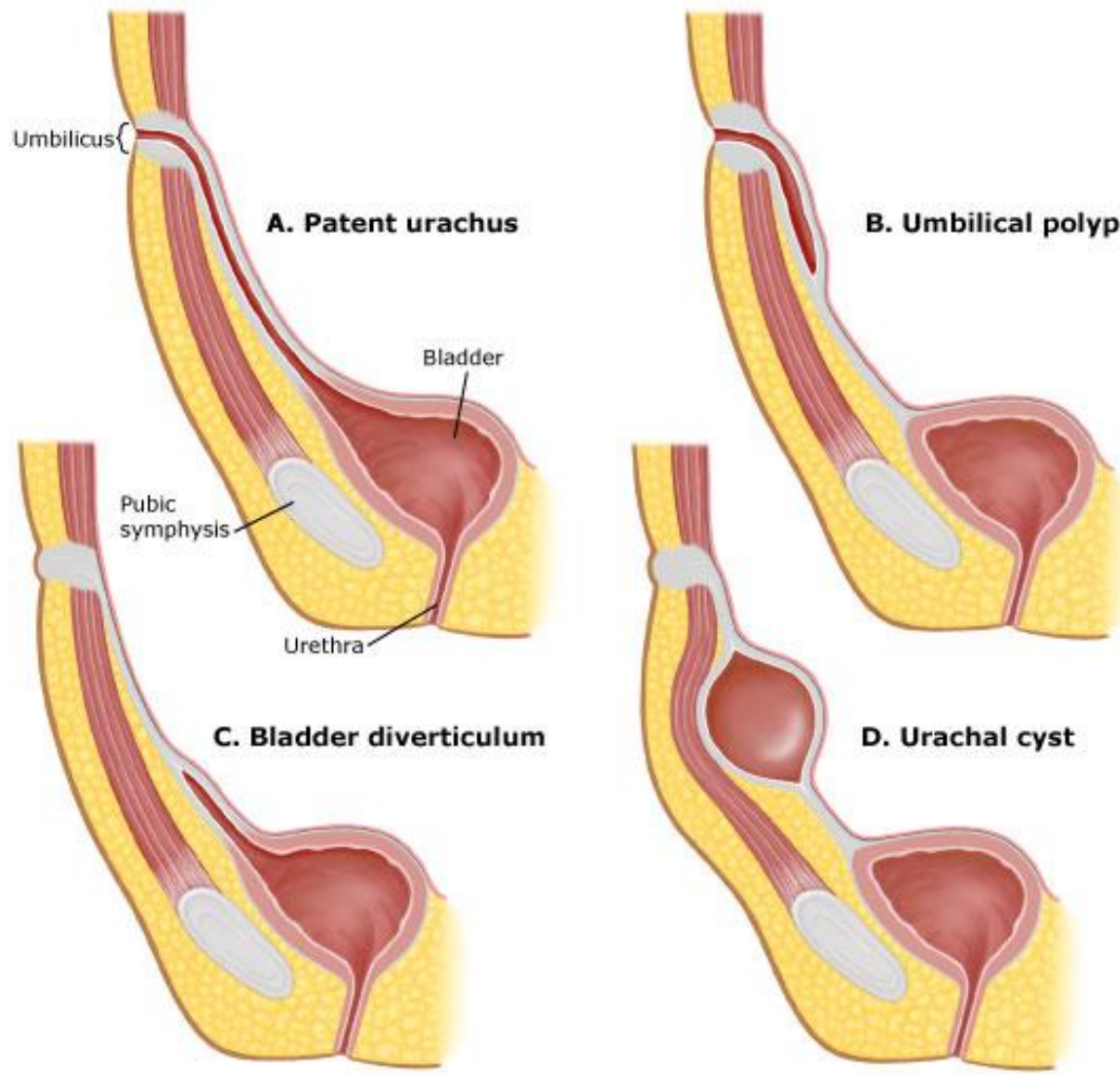

Figure 7: Diagram illustrating different types of urachal anomalies

The anomalies shown in Figure 6 are not exclusive as there can be combinations of the examples shown. For instance, there can be aurachal sinus associated with a urachal cyst. The relative incidence of the different types of urachal abnormalities from several clinical series are shown in Table 1.
Incidence: clinically important anomalies have an incidence of 2 in 300,000 births 5 . incidence of UC in adults is unknown but it is rare. It is more common in men than women2, 5 .

In a 31-year review, Risher et al ${ }^{2}$ found 12 adults with urachal anomalies, of which 5 were UC. 


\section{International Journal of Science and Research (IJSR) \\ ISSN (Online): 2319-7064 \\ Index Copernicus Value (2013): 6.14 | Impact Factor (2014): 5.611}

Table 1: Summary of the Types and Incidence of Urachal Anomalies presenting in children

\begin{tabular}{|c|c|c|c|c|c|}
\hline Author & \# Pts & Patent & Sinus & Cyst & Diverticulum \\
\hline Ashley & 46 & 6 & 14 & 25 & 1 \\
\hline Copp & 29 & 3 & 17 & 7 & 2 \\
\hline Cilento & 45 & 7 & 22 & 16 & 0 \\
\hline Galati & 23 & 2 & 9 & 12 & 0 \\
\hline Mesrobian & 21 & 2 & 9 & 9 & 1 \\
\hline Rich & 35 & 19 & 4 & 12 & 0 \\
\hline Yiee & 31 & 7 & 5 & 19 & 0 \\
\hline Total & 230 & $46(20 \%)$ & $80(35 \%)$ & $100(43 \%)$ & $4(2 \%)$ \\
\hline
\end{tabular}

Urachal diverticulum seems to be the most rare of the anomalies in all series, with some recent series not reporting any ${ }^{2-4,7-10}$.

Modes of presentation of urachal anomalies in adults differ from those seen in children. In adults, the commonest variety is urachal cyst, with infection being usual mode of presentation 2,5 .The route of infection is haematogenous, lymphatic, direct or ascending from the bladder

Bacteriology: The commonly cultured microorganisms from the cystic fluid include Escherichia coli, Enterococcus faecium, Klebsiella pneumonia, Proteus, Streptococcus viridans and Fusobacterium [5,6]. In our case, Bacteroidessp was cultured

Clinicalresentation

Most childhood urachal remnants will present with either fluid drainage from the umbilicus or a mass with evidence of infection. There is a bimodal age distribution with presentation at a mean of 1-3 months of age for those with aurachal sinus or patency versus a mean age of 3 years for those who present with a urachal cyst [7]. The characteristics of the drainage fluid are a clue to its cause. Persistent clear fluid leakage (likely urine) in an infant is highly suggestive of a patent urachus while cloudy, serous, or bloody fluid is more indicative of an urachal sinus or cyst.

The clinical signs and symptoms are non-specific, as UC are largely asymptomatic until they become infected. However, the presence of the triad of symptoms including a tender midline infraumbilical mass, umbilical discharge and sepsis should arouse suspicion of UC. If left untreated, UC slowly enlarges and may drain through the umbilicus as was seen in our patient, or drain into the bladder or both, resulting in alternating sinus. The common presenting symptoms for urachal anomalies are shown in Table 2.

Table 2: Presenting Symptoms in Children with Urachal Anomalies

\begin{tabular}{|c|c|c|c|c|c|c|}
\hline Author & \# Pts & Drainage & Pain & Mass/Infection & Asymptomatic & Other \\
\hline Cilento & 45 & 19 & 10 & 15 & 0 & 1 \\
\hline Copp & 29 & 13 & 0 & 10 & 5 & 1 \\
\hline Galati & 23 & 15 & 1 & 5 & 2 & 0 \\
\hline McCollum & 26 & 7 & 3 & 11 & 0 & 5 \\
\hline Mesrobian & 21 & 12 & 4 & 4 & 0 & 1 \\
\hline Yiee & 37 & 20 & 4 & 8 & 2 & 3 \\
\hline Total & $\mathbf{1 8 1}$ & $\mathbf{8 6}(\mathbf{4 8 \%})$ & $\mathbf{2 2 ( \mathbf { 1 2 \% } )}$ & $\mathbf{5 3 ( 2 9 \% )}$ & $\mathbf{9 ( 5 \% )}$ & $\mathbf{1 1 ( 6 \% )}$ \\
\hline
\end{tabular}

The differential diagnosis of umbilical drainage also includes

\section{Related Umbilical Disorders}

These result from incomplete closure of the omphalomesenteric duct.

\section{Omphalomesenteric duct.}

- This is extremely rare and may be recognized with fecal drainage noted from the umbilicus. It is more common in boys than in girls, and differentiation from urachal anomalies is important for the surgical approach. Confirmation is done through a fistulogram.

2. Partially patent omphalomesenteric duct.

A. Omphalomesenteric duct sinus.

B. Omphalomesenteric duct cyst.

This can be diagnosed with fistulograms and require excision.

3. Meckel's diverticulum.

- Persistence of the proximal portion of the omphalomesenteric duct as a diverticulum opening into the ileum is called a Meckel's diverticulum. It may be associated with an umbilical polyp.

4. Umbilical polyp.

- Persistence of intestinal mucosa at the umbilicus can develop into an umbilical polyp. Probing and possibly a fistulogram are important. A simple polyp can be treated superficially with silver nitrate or local excision. It is important, however, to make sure that it is not associated with a duct remnant.

5. Omphalocele.

- Failure of the intestines to recede into the abdominal cavity by the end of the tenth week of gestation results in an omphalocele.

- About $50 \%$ of infants with an omphalocele have other congenital anomalies.

6. Umbilical hernia.

- This is usually congenital and relates to the incomplete closure of the anterior abdominal wall fascia after the intestines have returned to the abdominal cavity.

7. Other.

A. Omphalitis

- Inflammation of the umbilicus

B. Single umbilical artery

- Controversy still exists over the single umbilical artery as a barometer of other congenital anomalies. Certainly, the incidence of urinary tract abnormalities is not significantly increased in newborns with a single umbilical artery.

These non-urachal causes need to be considered during the evaluation of patients with umbilical drainage.

\section{Investigations}

Ultrasound scan can help to make diagnosis in $77 \%$ of patients. ${ }^{6}$ Allen et al. ${ }^{8}$ and Ozbek et al. ${ }^{9}$ suggested that ultrasonography is an ideal modality for diagnosing urachal

\section{Volume 4 Issue 11, November 2015}




\section{International Journal of Science and Research (IJSR) \\ ISSN (Online): 2319-7064 \\ Index Copernicus Value (2013): 6.14 | Impact Factor (2014): 5.611}

cysts, since these entities are cystic, extraperitoneal, and are directly related to the bladder.

Nagasaki et al. ${ }^{10}$ reported a $75 \%$ diagnostic success rate for ultrasound, whereas Minevich et al. ${ }^{11}$ reported $57.1 \%$ and Cilento et al. $^{12}$ reported $100 \%$. However, cases of concomitant abscess and cellulitis were misinterpreted by ultrasonography. During primary diagnosis, ultrasonography, CT and MRI were performed to determine the size of the cyst and also the relationship between the peripheral tissue and the cyst. ${ }^{10}$

In the presence of an umbilical discharge, fistulography was performed to confirm the existence of a fistula, voiding cystourethrography was performed for reflux, ${ }^{13}$ and cystography was also carried out to investigate bladder cancer. In our case, ultrasound scan was not specific and MRI scan was used to make diagnosis and define the relationship to surrounding structures.

\section{Complications of Urachal Cyst}

- rupture into the peritoneal cavity leading to peritonitis.

- uracho-colonic fistula,

- stone formation and

- neoplastic transformation ${ }^{14-17}$.

The risk of urachal malignancy in adults is high and the prognosis is poor

\section{Treatment}

The treatment of choice for urachal cyst is by complete primary excision. An earlier report suggests a single stage procedure backed with appropriate antibiotic therapy for the treatment of infected UC ${ }^{13}$. However, Yoo et al ${ }^{6}$ in their study suggested a 2 stage procedure involving initial incision and drainage, followed by later excision of the urachal remnant.

The optimal treatment method for infected urachal cysts remains a subject of debate. The two-stage procedure produces a shorter postoperative hospital stay and no complications. However, in the case of small and localized infections, a single-stage excision can be considered in young adults without comorbidity. Thus, when evaluating complication frequencies alone, we determined that the twostage procedure presents a more-effective treatment option.

In our case, we adopted a staggered plan of management. Firstly, with administration of broad spectrum antibiotics guided by microbiology sensitivity, and after resolution of sepsis interval primary excision of the cyst.

\section{Conclusion}

Urachal anomalies are rare in adults. Presentation is atypical; therefore, a high index of suspicion is required in order to achieve a diagnosis. A triad of lower midline mass, umbilical discharge and sepsis is suggestive, although MRI confirms the diagnosis and defines the surrounding anatomical relationship. Complete surgical excision is the treatment of choice due to the risk of malignant transformation. We recommend a 2 stage treatment with a combination of broad spectrum antibiotics or incision and drainage, followed by interval excision after resolution of sepsis.

\section{References}

[1] Hammond G, Yglesias L, Davis JE: The urachus, its anatomy and associated fascia.Anat Rec 1941, 80:271274.

[2] Risher WH, Sardi A, Bolton J: Urachal abnormalities in adults: the Ochsner experience.South Med J 1990, 83:1036-1039.

[3] Begg RC: The Urachus: its Anatomy, Histology and Development .J Anat 1930, 64:170-183.

[4] MacNeily AE, Koleilat N, Kirulata HG, Homsey YL. Urachalabcesses: protean manifestations, thier recognition, and management. Urol 1992: 40:530-535.

[5] Ashley RA, Inman BA, Routh JC, Rohlinger AL, Husmann DA, Kramer SA: Urachal anomalies: a longitudinal study of urachal remnants in children and adults.JUrol 2007, 178:1615-16.

[6] Yoo KH, Lee SJ, Chang SG: Treatment of infected urachal cysts.Yonsei Med J 2006, 47:423-427.

[7] Yiee JH, Garcia N, Baker LA, et al. A diagnostic algorithm for urachal anomalies. J PediatrUrol 2007; 3:500-504.

[8] Allen JW, Song J, Velcek FT. Acute presentation of infected urachal cysts: case report and review of diagnosis and therapeutic interventions. PediatrEmerg Care. 2004;20:108-111.

[9] Ozbek SS, Pourbagher MA, Pourbagher A. Urachal remnants in asymptomatic children: gray-scale and color Doppler sonographic findings. J Clin Ultrasound. 2001;29:218-222.

[10] Nagasaki A, Handa N, Kawanami T. Diagnosis of urachal anomalies in infancy and childhood by contrast fistulography, ultrasound and CT. PediatrRadiol. 1991;21:321-323

[11] Minevich E, Wacksman J, Lewis AG, Bukowski TP, Sheldon CA. The infected urachal cyst: primary excision versus a staged approach. $\mathrm{J}$ Urol. 1997;157:1869-1872.

[12] Cilento BG, Jr, Bauer SB, Retik AB, Peters CA, Atala A. Urachal anomalies: defining the best diagnostic modality. Urology. 1998;52:120-122.

[13] Newman BM, Karp MP, Jeweet TC, Cooney DR. Advances in the management of infected urachal cysts. J Pediatr Surg. 1986;21:1051-1054.

[14] .Flanagan DA, Mellinger JD: Urachal-sigmoid fistula in an adult male. Am Surg 1998, 64:762-763

[15] Maruschke M, Kreutzer HJ, Seiter H: Bladder rupture caused by spontaneous perforation of an infected urachal cyst.Urologe A 2003, 42:834-839.

[16] Ohgaki M, Higuchi A, Chou H, Takashina K, Kawakami S, Fujita Y, Hagiwara A, Yamagishi $\mathrm{H}$ :Acute peritonitis caused by intraperitoneal rupture of an infected urachal cyst: report of a case. Surg Today 2003, 33:75-77.

[17] Mazzucchelli R, Scarpelli M, Montironi R: Mucinous adenocarcinoma with superficial stromal invasion and villous adenoma of urachal remnants: a case report. $J$ ClinPathol 2003, 56:465-467. 